\title{
Resiliencia y desempeño docente en tiempo de pandemia en instituciones
} educativas de secundaria peruana

\section{Resilience and teaching performance in times of pandemic in Peruvian secondary educational institutions}

\section{Blanca Yaneth Avila-Valdiviezo}

yanneavila06@gmail.com

Universidad César Vallejo, Chimbote

Perú

https://orcid.org/0000-0001-9090-5070

Carmen Elena Carbonell-García ccarbonellg@ucvvirtual.edu.pe

Universidad César Vallejo, Chimbote

Perú

https://orcid.org/0000-0002-3692-3013

Rosa María Salas-Sánchez

rsalas@ucvvirtual.edu.pe

Universidad César Vallejo, Chimbote

Perú

https://orcid.org/0000-0002-6454-8740

Recepción: 15 de marzo 2021

Revisado: 15 de mayo 2021

Aprobación: 15 de junio 2021

Publicación: 01 de julio 2021 


\title{
RESUMEN
}

La investigación de revisión documental tiene por objetivo analizar la resiliencia y desempeño docente en tiempo de pandemia en instituciones educativas de secundaria peruana. Entre los principales problemas laborales del docente es que labora desde el hogar y debe convivir con los problemas derivados de cada miembro de la familia, siendo importante advertir que emociones negativas como la depresión, soledad, ira, son contraproducentes para el buen desempeño profesional del docente. En consideración, la resiliencia incide favorablemente en la generación de un estado de satisfacción laboral por parte del docente, así mismo, se constituye en un agente protector a factores desencadenantes del estrés, ansiedad, depresión, como elementos adversos a la sanidad mental del profesional de la educación; siendo necesario considerar adaptar el currículo desde una concepción emocional - tecnológica con la finalidad de prever la minimización de situaciones adversas concomitantes a daños emocionales en el docente.

Descriptores: Salud mental; docente de secundaria; satisfacción en el trabajo; resiliencia. (Palabras tomadas del Tesauro UNESCO).

\begin{abstract}
The objective of the documentary review research is to analyze the resilience and teaching performance in times of pandemic in Peruvian secondary educational institutions. Among the main work problems of the teacher is that he works from home and must live with the problems derived from each member of the family, being important to note that negative emotions such as depression, loneliness, anger, are counterproductive for the good professional performance of the teacher . In consideration, resilience has a favorable impact on the generation of a state of job satisfaction by the teacher, likewise, it constitutes a protective agent against triggers of stress, anxiety, depression, as adverse elements to the mental health of the professional of The education; It is necessary to consider adapting the curriculum from an emotionaltechnological conception in order to foresee the minimization of adverse situations concomitant to emotional damage to the teacher.
\end{abstract}

Descriptors: Mental health; secondary school teachers; job satisfaction; resilience. (Words taken from the UNESCO Thesaurus). 


\section{INTRODUCCIÓN}

La pandemia por COVID-19 no solo ha generado problemas sanitarios a nivel de quienes se han contagiado con el virus, sino, que como se inició el proceso de transmisión de forma rápida a nivel mundial, además sin contar con un antecedente de como era la cadena de transmisión y contagio, generó alertas emocionales en la población, sumado al período de confinamiento, el cual promovió cambios conductuales en las personas, siendo algunos de ellos "hacer frente a la depresión, la ansiedad y el trauma emergentes, y el aumento de los conflictos relacionales" (Fraenkel \& Cho, p. 1).

De ese modo, quienes han demostrado ser resilientes a la adversidad han transitado con mayor firmeza este período crítico de pandemia desde una óptica emocional (Hallal, 2020). En este sentido, se debe comprender que "la resiliencia predice una respuesta positiva a la situación estresante (la experiencia de emociones positivas) mientras que el afrontamiento puede ser positivo (solucionando el problema) o negativo (negando la situación)" (Ortunio, et al. 2016, p. 99). Por lo tanto, es un modo de abordar la realidad sin huir de ella, evadiéndola, si no, afrontándola como motivación a superarla desde la concepción que siempre existirán mejores oportunidades para la persona.

En complemento, (Oriol-Bosch, 2012), comenta que el ser resiliente, se asocia al hecho de buscar predictores emocionales favorables que contribuyan a articular un posicionamiento de la persona frente a la adversidad en condiciones de asumir con una aptitud agradable con la intención de bloquear las respuestas negativas como agresividad, ira, desencadenantes de estados emocionales no favorables para el afrontamiento individual - social de los problemas; lo cual, en palabras de (Pastor, 2021), tendría relación con una conducta apegada a la ética, procurando en el tiempo de pandemia, priorizar la adquisición de bienes y servicios, así como promover la templanza como una acción bioética favorable para compaginar un comportamiento resiliente en el quehacer diario de la persona.

Por otro lado, (Russell, 2021), destaca la importancia de identificar como comunidad aspectos que permitan trabajar cooperativamente para superar las adversidades sociales 
derivadas de la pandemia, siendo esto, un indicador de resiliencia colectiva por cuanto se definen aspectos de comportamiento desde la lucha común por sobrevivir en medios del caos sanitario, por lo que podría relacionarse la resiliencia con las ganas de vivir, pero sobre todo de optimizar los recursos con los cuales se cuenta para conquistar un mejor estilo de vida en medio de la crisis.

A partir de lo develado, la investigación se plantea una revisión del estado de la cuestión sobre el tema de resiliencia y desempeño docente durante el periodo de confinamiento por COVID-19, empleándose para tal fin, el método analítico sintético y la técnica de análisis de contenido con la intención de proyectar una síntesis teórica sobre el tema, a partir de escrutar trabajos de investigación publicados en Pubmeb, Scielo, Scopus, WOS, Latindex 2.0, REDALYC, así como repositorios institucionales de organismos y universidades públicas - privadas.

De ese modo, la investigación de revisión documental tiene por objetivo analizar la resiliencia y desempeño docente en tiempo de pandemia en instituciones educativas de secundaria peruana.

\section{Resiliencia y desempeño docente en tiempo de pandemia en instituciones educativas de secundaria peruana:}

Es así que la pandemia por COVID - 19 no solo ha afectó el aspecto psicológico emocional de las personas, sino, que alteró su socialización, resultando la educación como un área de mayor afectación, por cuanto se trastocó el compartir en un aula de forma presencial, sino, que se alteró el paradigma de trabajo para los docentes, producto de implementar la virtualidad en su totalidad para tratar de proseguir con la formación de los estudiantes, destacándose que la población docente peruana es heterogénea en la implementación de las TIC, aunado a los problemas de conexión y de equipación tecnológica requerida para laborar óptimamente, siendo esto en igualdad de condiciones para el sector estudiantil, aunque los estudiantes se encontraban más habituados a lo tecnológico, proyectando estrés en los docentes al tener que diseñar contenidos 
pedagógicos a través de la virtualidad que cumpliesen con las expectativas de los estudiantes (Pizan-Campos, et al. 2020).

En complemento, el estudio de (Santos, et al. 2020), advierte sobre una percepción negativa por parte de los docentes al proceso de educación en período por confinamiento en el Perú, destacando el poco involucramiento de las familias, así como evidencian preocupación por cuanto consideran que los estudiantes no se han formado diligentemente, producto de las barreras y limitaciones al enfoque educativo de la virtualidad, empleándose con mayor predominancia el WhatsApp como medio o recurso para la transmisión de actividades educativas. Ante lo cual, hay que tener en cuenta que:

El hacinamiento impide contar con un espacio adecuado para estudiar y descansar, lo que repercute en el desarrollo cognitivo en la infancia y las trayectorias laborales y de bienestar en la adultez, a la vez que favorecen una mayor propensión a situaciones de abuso (CEPAL-UNESCO, 2020, p 14).

Lo planteado por la CEPAL-UNESCO, no solo afecta el rendimiento escolar del estudiante, sino, el laboral del docente, proyectando estrés, tensión, por cuanto implica convivir en el hogar con los problemas derivados de cada miembro de la familia, más el propio producto de estar laborando virtualmente, viéndose compartidos roles dentro de un mismo espacio habitacional sin el adecuado tiempo o área para el requerido esparcimiento, viéndose afectada la salud emocional del docente, lo cual, podría influir negativamente su desempeño profesional, siendo conveniente tener presente el antecedente de (Revilla-Mendoza \& Palacios-Jiménez, 2020), donde se indica que los estudiantes valoraron en rango medio de productividad a los docentes.

Destacándose, el trabajo de (Huamán-Ramos, et al. 2021), al advertir que emociones negativas como la depresión, soledad, ira, son contraproducentes para el buen desempeño profesional del docente, siendo conveniente a partir de lo planteado, estudiar como la resiliencia podría influir en superar los inconvenientes descritos anteriormente, así, como podría contribuir en el mejoramiento de los docentes desde una óptica personal, social, pedagógica. 
Así mismo, la investigación de (Aldana-Zavala \& Rodríguez-Partidas, 2020), confirman la presencia de estrés, depresión y ansiedad en docentes durante el periodo de confinamiento por COVID-19, siendo estos elementos que deben ser abordados con ayuda especializada con la finalidad de evitar el desarrollo de cuadros sanitarios adversos desde el punto de vista psicológico - psiquiátrico, además que tal acompañamiento contribuye a mejorar la perspectiva de vida para ser resiliente ante el escenario adverso presentando durante la pandemia.

Desde esa variante, (García-Cedeño, et al. 2020), plantean que es necesario promover un currículo de emergencia donde sea posible integrar el cuidado de la salud mental de los docentes como un aporte a su seguridad social, de ese modo, se ventila confianza y apoyo para establecer mayores vínculos de fidelidad, confiabilidad, propiciando un clima organizacional favorable a establecer un espíritu resiliente en coordinación de promover un mejor desempeño docente en el accionar profesional pedagógico que les toca desempeñar a la luz de un periodo incierto donde no se proyecta una fecha tangible para el fin de la pandemia.

Lo cual contribuye a la necesidad de contar con políticas educativas conectadas con otras áreas prioritarias como la salud, alimentación, para favorecer a los docentes a contar con una mejor atención integral para su bienestar social, lo cual, puede contribuir en establecer un estado motivacional para afrontar los diversos desafíos que en materia de atención pedagógica se requieren para establecer un desempeño profesional de mayor efectiva para la formación de los estudiantes sin riesgo al detrimento de la calidad educativa y de la salud mental del docente (Inga-Paida, et al. 2020).

Otro aspecto a tener en consideración para la mejora del desempeño profesional docente, es lo referente al equipamiento tecnológico, por cuanto es fundamental para contar con una formación de calidad a través de la virtualidad, siendo necesario involucrar en la legislación peruana (Quezada-Castro, et al. 2021), por cuanto se complementa en reconocer el valor del docente para la sociedad, siendo un estimulo que contribuye a ser 
resiliente en la medida que percibe que las adversidades son momentáneas en razón de establecer una nueva adaptabilidad social.

Entre los elementos a tener en consideración para el uso obligatorio por parte del docente, se presentan en la figura 1, por lo tanto, al no contar con los mismos en optimas condiciones, apelará a la resiliencia como factor que contribuya a fortalecer su motivación en procura de cumplir con sus obligaciones y no desmejorar su rendimiento profesional.

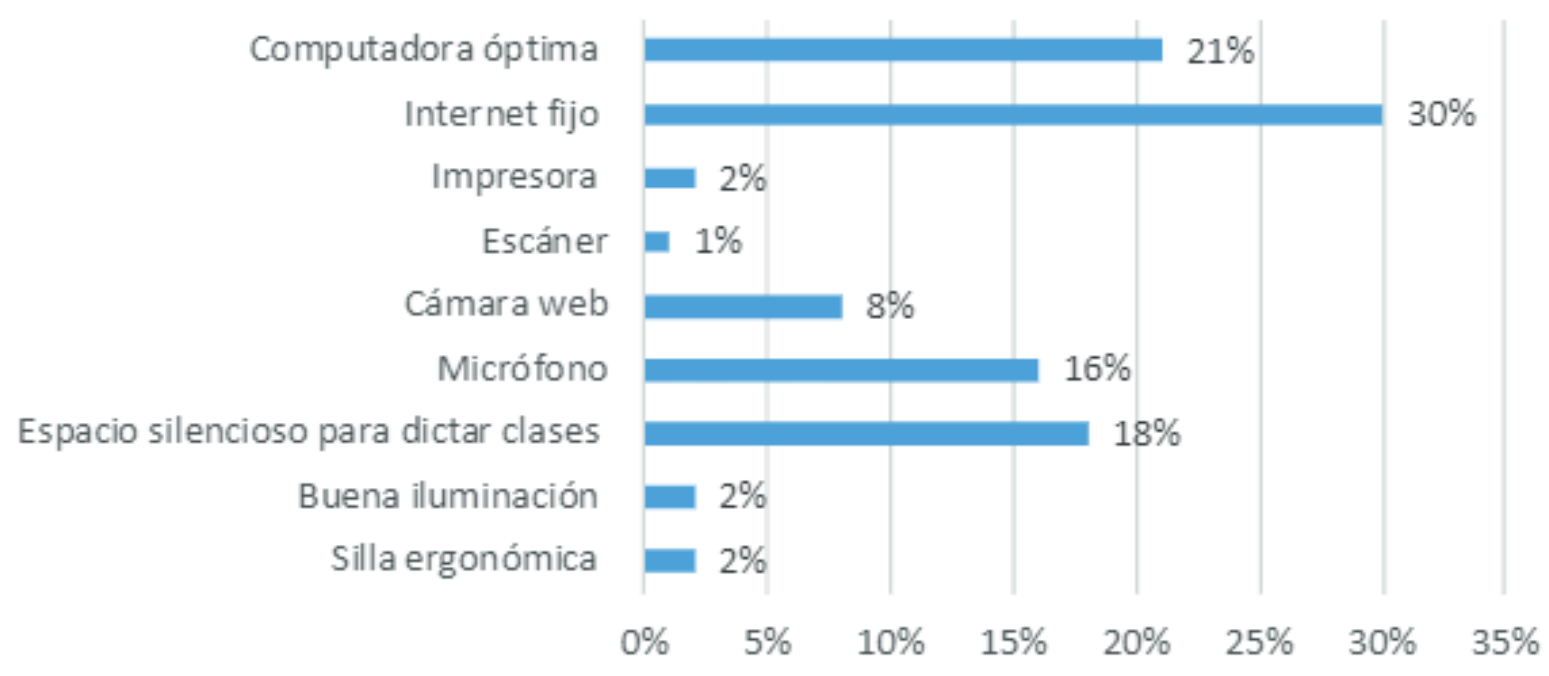

Figura 1. ¿Qué condiciones laborales son necesarias para el desarrollo de la docente en los entornos virtuales de aprendizaje?

Fuente: Quezada-Castro, et al. (2021).

La figura 1, presenta nueve elementos que son necesarios para la conformación de un clima laboral para el docente en condiciones favorables, destacándose además que se debe establecer la posibilidad de contar con un espacio favorable para la transmisión de las clases, por cuanto se puede correr el riesgo de tener impedimentos al tener que compartir el rol del docente con el de padre o madre por ejemplo, si se encuentra en la casa de habitación para establecer su desempeño laboral, siendo una acción resiliente el no quebrarse emocionalmente en su accionar laboral y del hogar. 
En complemento de lo planteado, (Apaza, et al. 2020), explican la necesidad de promover factores psicosociales favorables por cuanto esto implica mayor posibilidad de que el docente eleve su productividad, como se ha mencionado a lo largo del trabajo, es necesario promover condiciones laborales óptimas por cuanto además, permite tener mayor posibilidad de manejar asertivamente los conflictos emocionales propios de un estado de confinamiento, así como de trabajar desde casa donde otros miembros de la familia también están trabajando o estudiando, teniendo en cuenta que si el docente tiene hijos en edad escolar, deberá establecer un tiempo para acompañarlos.

Es allí donde este acompañamiento puede contribuir a comprender la perspectiva de sus colegas, e incluso de los propios estudiantes, posibilitando contar con empatía como medio para proyectar una sana socialización desde los espacios virtuales de aprendizaje, siendo desde la posición de (Castagnola-Sánchez, et al. 2021), contar con:

a) Un currículo socio emocional, en el que los directivos, el maestro, los padres estén involucrado y comprometidos con el desarrollo de la resiliencia, la autoestima, la motivación de los estudiantes.

b) Evaluaciones psicológicas y posteriores terapias integrales de ser necesarias, con el fin de soslayar pensamientos automáticos negativos, catastróficos y pesimistas sobre el futuro.

De ese modo, se procura promover una salud emocional para evitar el desgaste del docente a pesar de que pueda ser resiliente, por cuanto podría verse amenazado con la aparición del síndrome de Burnout, dado que este se relaciona con altos niveles de estrés y percepción negativa en la calidad de vida del trabajador (Vidotti, et al. 2019), siendo estos elementos contrarios a la resiliencia, por lo que se proyecta que si el docente se ve envuelto en el desgaste laboral, su productividad descenderá notablemente en su desempeño profesional, sobre todo porque:

El Síndrome de Burnout se manifiesta cuando un trabajador que antes estaba muy implicado afectivamente y efectivamente con el proceso de producción referente a su ocupación, ya sea con sus clientes o con el trabajo en sí, pasa a sufrir estrés continuo y excesivo desgaste y no consigue distinguirlo de la fatiga (Almeida-Freire, et al. 2015, p. 20). 
En ese sentido, el accionar resiliente contribuye a detectar estados de ánimos no favorables o habituales en la persona, posibilitando el acompañamiento profesional con la finalidad de establecer un vinculo de atención emocional en favor de articular un trabajo favorable desde el fortalecimiento de los lazos emocionales no solo con el trabajo, sino, consigo mismo, proyectando una socialización favorable en favor de articular un desenvolvimiento profesional acorde a las exigencias educativas de la educación virtual. Partiendo de lo planteado, (Yncera-Hernández, et al. 2021), invitan a fortalecer la resiliencia en los docentes como actores educativos fundamentales en el proceso de aprendizaje, para lo cual, consideran necesario la aplicación de técnicas de autocuidado emocional con la intención de favorecer el manejo asertivo de los factores que puedan ocasionar estrés, depresión, contribuyendo a desencadenar circunstancias adversas para la salud emocional y por ende productiva del trabajador educativo.

Por otro lado, (Medina, et al. 2020), confirman que las personas resilientes, tienen mayor posibilidad de superar las adversidades emocionales, sociales, producidas por la pandemia COVID-19, en referencia a la educación, debido que permite un afrontamiento positivo de la realidad, adaptándose al escenario y aprovechando lo negativo como una oportunidad para crecer como persona, especialmente si se aprovecha para educar a ser feliz, lo cual se puede interpretar como una educación emocional (Ángeles-Donayre, M., \& Manrique-Tapia, 2021).

En consecuencia, es necesario redimensionar la educación desde su currículo, entendiéndose como el todo de la organización, por cuanto no se puede exigir al docente que labore efectivamente si no se le brinda el debido acompañamiento emocional y social para tal fin; siendo indispensable redefinir el como trabajan las organizaciones para actuar a la luz de las exigencias de la sociedad digital (García-Madurga, et al. 2021). El trabajo de (Díaz-Sánchez \& Barra-Almagiá, 2017), indica un elemento a tener en consideración cuando se estudia la resiliencia y la satisfacción docente, en este caso, hacen referencia a la antigüedad del servicio, destacan que entre mayor experiencia es posible que el docente se sienta con mayor seguridad y pueda disfrutar del trabajo, 
propiciándose un estado resiliente para lograr superar adversidades, aunque debido al cambio brusco de modalidad de estudio donde se pasó de lo presencial a lo virtual, se ha generado estrés por no tener claridad en principio de como manejar todos los programas que brinda el mundo digital - tecnológico.

En complemento, Palomeque \& Parrales (2021), indican que "se ha podido determinar que el aumento de la satisfacción laboral va de la mano con una adaptación positiva al medio" (p. 312). De ese modo, entre más rápido se genere la aceptación por parte del docente que se encuentra frente a un nuevo paradigma laboral, para el cual debe adaptarse sobre la marcha de los acontecimientos, se generará mayor resiliencia contribuyendo a una mejor satisfacción en el trabajo, evitándose la prolongación del estrés que podría conducir al síndrome de Burnout (Vicente-de-Vera-García \& GabariGambarte, 2019).

\section{CONCLUSIÓN}

La resiliencia incide favorablemente en la generación de un estado de satisfacción laboral por parte del docente, así mismo, se constituye en un agente protector a factores desencadenantes del estrés, ansiedad, depresión, como elementos adversos a la sanidad mental del profesional de la educación; siendo necesario considerar adaptar el currículo desde una concepción emocional - tecnológica con la finalidad de prever la minimización de situaciones adversas concomitantes a daños emocionales en el docente, siendo considerable establecer tales parámetros en la contratación de los docentes con la intención de promover un clima organizacional favorable, sobre todo, cuando este ha sido desarrollado en la casa de habitación como medida preventiva por la pandemia, de ahí, que se deben estudiar aspectos relacionados a la calidad de vida del docente para lograr verdaderamente una motivación positiva a transcender desde la resiliencia las diversas adversidades profesionales. 


\section{FINANCIAMIENTO}

No monetario.

\section{AGRADECIMIENTO}

A la Universidad César Vallejo por el apoyo a la investigación.

\section{REFERENCIAS CONSULTADAS}

Aldana-Zavala, J., \& Rodríguez-Partidas, N. (2020). Depresión, ansiedad y estrés por COVID -19 en actores educativos [Depression, anxiety and stress due to COVID 19 in educational actors]. Revista Arbitrada Interdisciplinaria de Ciencias de la Salud. Salud y Vida, 4(8), 6-23. http://dx.doi.org/10.35381/s.v.v4i8.932

Almeida-Freire, M, Oliveira, E, Guimarães Ximenes Neto, F, Evangelista Lopes, R, Gomes Nogueira Ferreira, A, \& Vieira Gomes, B. (2015). Síndrome de Burnout: un estudio con profesores [Burnout syndrome: a study with teachers]. Salud de los Trabajadores, 23(1), 19-28.

Ángeles-Donayre, M., \& Manrique-Tapia, C. (2021). La resiliencia como herramienta de cambio para alcanzar la felicidad en adolescentes de Lima Norte [Resilience as a tool for change to achieve happiness in adolescents in North Lima]. PsiqueMag, 10(1), 90-97.

Apaza P., C. M., Seminario Sanz, R. S., \& Santa-Cruz Arévalo, J. E. (2020). Factores psicosociales durante el confinamiento por el Covid-19 - Perú [Psychosocial factors during confinement by Covid-19 - Peru]. Revista Venezolana De Gerencia, 25(90), 402-413. https://doi.org/10.37960/rvg.v25i90.32385

Castagnola-Sánchez, C, Carlos-Cotrina, J, \& Aguinaga-Villegas, D. (2021). La resiliencia como factor fundamental en tiempos de Covid-19 [Grief and resilience as a fundamental factor in times of Covid-19]. Propósitos y Representaciones, 9(1), e1044. https://dx.doi.org/10.20511/pyr2021.v9n1.1044

CEPAL-UNESCO. (2020). La educación en tiempos de la pandemia de COVID-19 [Education in times of the COVID-19 pandemic]. http://hdl.handle.net/11362/45904 
Díaz-Sánchez, C, \& Barra-Almagiá, E. (2017). Resiliencia y satisfacción laboral en profesores de colegios municipales y particulares subvencionados de la comuna de Machalí [Resilience and job satisfaction among teachers from public and private subsidized schools from the commune of Machalí]. Estudios pedagógicos (Valdivia), 43(1), 75-86. https://dx.doi.org/10.4067/S0718-07052017000100005

Fraenkel, P., \& Cho, W. L. (2020). Reaching Up, Down, In, and Around: Couple and Family Coping During the Coronavirus Pandemic. Family process, 59(3), 847-864. https://doi.org/10.1111/famp.12570

García-Cedeño, G., Vélez-Loor, M., Franco-Zambrano, C., \& Ormaza-Bermello, M. (2020). Educación por competencias: Una posibilidad reordenadora del currículo pensada en emergencia escolar por COVID-19 [Education by competencies: A possibility to rearrange the curriculum thought about a school emergency due to COVID-19]. EPISTEME KOINONIA, 223-237. http://dx.doi.org/10.35381/e.k.v3i5.770

García-Madurga, M, Grilló-Méndez, A, \& Morte-Nadal, T. (2021). La adaptación de las empresas a la realidad COVID: una revisión sistemática [The adaptation of companies to the COVID reality: a systematic review]. RETOS. Revista de Ciencias de la Administración y Economía, 11(21), 5570. https://doi.org/10.17163/ret.n21.2021.04

Hallal, P. C. (2020). Resistance and Resilience in the throes of a Pandemic. Resistência e resiliência em tempos de pandemia. Ciencia \& saude coletiva, 25(9), 3342. https://doi.org/10.1590/1413-81232020259.20312020

Huamán-Ramos, L.., Torres Inga, L. A., Amancio Anzuhueldo, A. M. \& Sánchez Díaz, S. (2021). Educación remota y desempeño docente en las instituciones educativas de Huancavelica en tiempos de COVID-19 [Remote education and teaching performance in educational institutions of Huancavelica in times of COVID19]. Apuntes Universitarios, 11(3), 45 - 59. https://doi.org/10.17162/au.v11i3.692

Inga-Paida, M., Garcia-Herrera, D., Castro-Salazar, A., \& Erazo-Álvarez, J. (2020). Educación y Covid-19: Percepciones docentes para enfrentar la pandemia [Education and Covid-19: Teachers' perceptions to face the pandemic]. Revista Arbitrada Interdisciplinaria Koinonía, 5(1), 310-331. http://dx.doi.org/10.35381/r.k.v5i1.785 


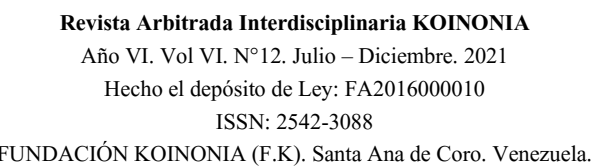

Blanca Yaneth Avila-Valdiviezo; Carmen Elena Carbonell-García; Rosa María Salas Sánchez

Medina, G., Lujano, Y., Aza, P., \& Sucari, W. (2020). Resiliencia y engagement en estudiantes universitarios durante el contexto del COVID 19 [Resilience and engagement in university students during the context of COVID 19]. Revista Innova Educación, 2(4), 658-667. https://doi.org/10.35622/j.rie.2020.04.010

Oriol-Bosch, A. (2012). Resiliencia [Resilience]. Educación Médica, 15(2), 77-78.

Ortunio C, Magaly S, \& Guevara R, Harold. (2016). Apróximación teórica al constructo resiliencia [Theoretical approach to the resilience construct]. Comunidad $y$ Salud, 14(2), 96-105.

Palomeque, M., \& Parrales, M. (2021). La resiliencia y satisfacción laboral de docentes en una institución educativa de la ciudad de Guayaquil [Resilience and job satisfaction of teachers in an educational institution in the city of Guayaquil]. 593 Digital Publisher CEIT, 6(1), 303-317. https://doi.org/10.33386/593dp.2021.1.427

Pastor L. M. (2021). Nota del Editor: COVID-19: resiliencia y temple éticos [Editor's Note: COVID-19: resilience and temperance]. Cuadernos de bioetica: revista oficial de la Asociación Española de Bioética y Ética Medica, 32(104), 11-14. https://doi.org/10.30444/CB.83

Pizan-Campos, E, Barros-Sevillano, S, \& Yupari-Azabache, I. (2020). Impacto del COVID19 en la educación de los estudiantes de medicina del Perú [Impact of COVID-19 on the education of medical students in Peru]. Revista de la Facultad de Medicina Humana, 20(3), 534-535. https://dx.doi.org/10.25176/rfmh.v20i3.2959

Quezada-Castro, M. del P., Castro Arellano, M. del P., Dios Castillo, C. A., \& Quezada Castro, G. A. (2021). Condiciones laborales en la educación universitaria peruana: Virtualización ante la pandemia COVID -19 [Working conditions in Peruvian university education: Virtualization in the face of the COVID -19 pandemic]. Revista Venezolana De Gerencia, 26(93), 110-123. https://doi.org/10.52080/rvg93.09

Revilla-Mendoza, J. T., \& Palacios-Jiménez, A. S. (2020). Ser docente en tiempos de pandemia por covid-19: evaluación del desempeño docente en una universidad pública de Lima [Being a teacher in times of a covid-19 pandemic: evaluation of teaching performance at a public university in Lima]. Revista Científica Ágora, 7(2), 58-62. https://doi.org/10.21679/arc.v7i2.197 
Russell C. (2021). Supporting community participation in a pandemic. Gaceta sanitaria, S0213-9111(21)00010-8. Advance online publication. https://doi.org/10.1016/j.gaceta.2021.01.001

Santos, V., Villanueva, I., Rivera, E., \& Vega, E. (2020). Percepción docente sobre la educación a distancia en tiempos de COVID-19 [Teachers' perception of distance education in times of COVID-19]. CienciAméRica, 9(3), 126-141. http://dx.doi.org/10.33210/ca.v9i3.352

Vicente-de-Vera-García, I. \& Gabari-Gambarte, I. (2019). La resiliencia como factor protector del estrés crónico en docentes [Resilience as a protective factor of chronic stress in teachers]. European Journal of Investigation in Health Psychology and Education, 9(3), 159-175.

Vidotti, V, Martins, J, Galdino, M, Ribeiro, R, \& Robazzi, M. (2019). Síndrome de burnout, estrés laboral y calidad de vida en trabajadores de enfermería [Burnout syndrome, work stress and quality of life in nursing workers]. Enfermería Global, 18(55), 344376.

Yncera-Hernández, N, Lorenzo Ruiz, A, \& Peña Salazar, L. (2021). Proyecto para el desarrollo y fortalecimiento del proceso de resiliencia en una comunidad educativa ante la pandemia de COVID-19 [Project for the development and strengthening of the resilience process in an educational community facing COVID-19 pandemic]. Dilemas contemporáneos: educación, política y valores, 8(spe1), 00005. https://doi.org/10.46377/dilemas.v8i.2559

C2021 por los autores. Este artículo es de acceso abierto y distribuido según los términos y condiciones de la licencia Creative Commons Atribución-NoComercial-Compartirlgual 4.0 Internacional (CC BY-NC-SA 4.0) (https://creativecommons.org/licenses/by-nc-sa/4.0/). 